Original Research Paper

\title{
Hybrid Block Method for Direct Numerical Approximation of Second Order Initial Value Problems Using Taylor Series Expansions
}

\author{
Oluwaseun Adeyeye and Zurni Omar \\ Department of Mathematics, School of Quantitative Sciences, Universiti Utara Malaysia, Sintok, Kedah, Malaysia
}

Article history

Received: 26-11-2016

Revised: $14-02-2017$

Accepted: 15-02-2017

Corresponding Author:

Oluwaseun Adeyeye

Department of Mathematics,

School of Quantitative

Sciences, Universiti Utara

Malaysia, Sintok, Kedah,

Malaysia

Email:

adeyeye_oluwaseun@ahsgs.uum.edu.my

\begin{abstract}
In this article, a hybrid block method is utilized for the numerical approximation of second order Initial Value Problems (IVPs). The rigor of reduction to a system of first order initial value problems is bypassed as the hybrid block method directly solves the second order IVPs. Likewise, the methodology utilized also avoids the cumbersome steps involved in the widely adopted interpolation approach for developing hybrid block methods as a simple and easy to implement algorithm using the knowledge from the conventional Taylor series expansions with less cumbersome steps is introduced. To further justify the usability of this hybrid block method, the basic properties which will infer convergence when adopted to solve differential equations are investigated. The hybrid block method validates its superiority over existing methods as seen in the improved accuracy when solving the considered numerical examples.
\end{abstract}

Keywords: Hybrid Block Method, Second Order, Direct Methods, Initial Value Problems, Taylor Series

\section{Introduction}

Differential equations of the form:

$$
\frac{d^{2} y}{d x^{2}}=f\left(x, y, \frac{d y}{d x}\right), y(a)=y, \frac{d y}{d x}(a)=y_{\mathrm{o}}^{\prime}, x \in[a, b]
$$

Are known as second order initial value problems with initial conditions prescribed at a certain point $a$. Differential equations of this form play an important role in modeling virtually every physical or biological process because such equations occur in connection with numerous problems that are encountered in various aspects of our everyday life. This concept of mathematical modeling involves translating problems from an application area into tractable mathematical formulations whose theoretical and numerical analysis provide insight, answers and guidance useful for the originating application (Abdelrahim and Omar, 2016; Moaddy et al., 2015).

Conventionally, the approach for obtaining a numerical approximation to equations of the form (1) above (also classified as higher order ordinary differential equations) usually involves a rigorous reduction of the higher order differential equations to a system of first order differential equations (James et al., 2013; Kayode and Adeyeye, 2011; Jator, 2007). Block multistep methods have evolved with time for the direct solution of second order initial value problems (Abdelrahim and Omar, 2016; Jator, 2007; Adesanya et al., 2012) and the concept of introducing evaluation at offgrid points birthing the introduction of hybrid block methods for numerical approximation of (1) have also been investigated into.

However, research continually improves upon previous by introducing new methods with better accuracy for numerically approximating differential equations. Hence, the main objective of this article which is the introduction of a new hybrid block method for numerically approximating (1) with better accuracy when compared to previously existing methods in terms of error. The methodology for developing the hybrid method is a new and easy to understand apptroach which differs from the commonly used interpolation (Jator, 2007; Adesanya et al., 2012; Kuboye and Omar, 2015) and numerical integration approach (See et al., 2016; Abdul Majid et al., 2012; Omar and Suleiman, 2016) for developing multistep methods.

\section{Materials and Methods}

The hybrid block method scheme for the direct numerical solution of (1) is written as: 
$y_{n+\frac{5}{6} \xi}=\sum_{i=0}^{1} \frac{\left(\frac{5}{6} \xi h\right)^{i}}{i !} y_{n}^{(i)}+h^{2} \sum_{i=0}^{6} \phi_{\xi i} f_{n+\frac{5}{6} i}, \xi=1,2, \cdots, 6$

Recall that from the form of Equation 1 above, it is likewise needed to have an expression that computes the derivative, which is $y^{\prime}$ of the second order initial value problem under consideration. This is obtained from the block scheme defined below:

$$
y_{n+\frac{5}{6} \xi}^{\prime}=y_{n}^{\prime}+h \sum_{i=0}^{6} \omega_{\xi i} f_{n+\frac{5}{6} i}, \xi=1,2, \cdots, 6
$$

Having defined the schemes in Equation 2 and 3 above, the next step is computing the values of the coefficients $\phi_{\xi i}$ and $\omega_{\xi i}$. This is derived by adopting the concept of derivation of linear multistep methods through Taylor expansions which dates back to the work of (Lambert, 1973). The approach considered the Taylor expansion for $y_{n+a}=y\left(x_{n}+a h\right)$ about $x_{n}$ defined as:

$y\left(x_{n}+a h\right)=y\left(x_{n}\right)+a h y^{(1)}\left(x_{n}\right)+\frac{(a h)^{2}}{2 !} y^{(2)}\left(x_{n}\right)+\cdots$

Where:

$$
\begin{aligned}
& y^{(q)}\left(x_{n}\right)=\left.\frac{d^{q} y}{d x^{q}}\right|_{x=x_{n}}, q=1,2, \cdots \\
& y_{n+\frac{5}{6}}=\sum_{i=0}^{1} \frac{\left(\frac{5}{6} h\right)^{i}}{i !} y_{n}^{(i)}+h^{2} \sum_{i=0}^{6} \phi_{1 i} f_{n+\frac{5}{6} i} \\
& \Rightarrow y\left(x_{n}\right)+\frac{5}{6} h y^{(1)}\left(x_{n}\right)+\frac{\left(\frac{5}{6} h\right)^{2}}{2 !} y^{(2)}\left(x_{n}\right)+\frac{\left(\frac{5}{6} h\right)^{3}}{3 !} y^{(3)}\left(x_{n}\right)+\frac{\left(\frac{5}{6} h\right)^{4}}{4 !} y^{(4)}\left(x_{n}\right)+\frac{\left(\frac{5}{6} h\right)^{5}}{5 !} y^{(5)}\left(x_{n}\right) \\
& +\frac{\left(\frac{5}{6} h\right)^{6}}{6 !} y^{(6)}\left(x_{n}\right)+\frac{\left(\frac{5}{6} h\right)^{7}}{7 !} y^{(7)}\left(x_{n}\right)+\frac{\left(\frac{5}{6} h\right)^{8}}{8 !} y^{(8)}\left(x_{n}\right)=y_{n}+\frac{5}{6} h y_{n}^{(1)}+h^{2}\left\{\phi_{10}\left(y^{(2)}\left(x_{n}\right)\right)\right. \\
& +\phi_{11}\left(y^{(2)}\left(x_{n}\right)+\frac{5}{6} h y^{(3)}\left(x_{n}\right)+\frac{\left(\frac{5}{6} h\right)^{2}}{2 !} y^{(4)}\left(x_{n}\right)+\frac{\left(\frac{5}{6} h\right)^{3}}{3 !} y^{(5)}\left(x_{n}\right)+\frac{\left(\frac{5}{6} h\right)^{4}}{4 !} y^{(6)}\left(x_{n}\right)\right. \\
& \left.+\frac{\left(\frac{5}{6} h\right)^{5}}{5 !} y^{(7)}\left(x_{n}\right)+\frac{\left(\frac{5}{6} h\right)^{6}}{6 !} y^{(8)}\left(x_{n}\right)\right)+\phi_{12}\left(y^{(2)}\left(x_{n}\right)+\frac{10}{6} h y^{(3)}\left(x_{n}\right)+\frac{\left(\frac{10}{6} h\right)^{2}}{2 !} y^{(4)}\left(x_{n}\right)\right. \\
& \left.+\frac{\left(\frac{10}{6} h\right)^{3}}{3 !} y^{(5)}\left(x_{n}\right)+\frac{\left(\frac{10}{6} h\right)^{4}}{4 !} y^{(6)}\left(x_{n}\right)+\frac{\left(\frac{10}{6} h\right)^{5}}{5 !} y^{(7)}\left(x_{n}\right)+\frac{\left(\frac{10}{6} h\right)^{6}}{6 !} y^{(8)}\left(x_{n}\right)\right)+\phi_{13}\left(y^{(2)}\left(x_{n}\right)\right. \\
& +\frac{15}{6} h y^{(3)}\left(x_{n}\right)+\frac{\left(\frac{15}{6} h\right)^{2}}{2 !} y^{(4)}\left(x_{n}\right)+\frac{\left(\frac{15}{6} h\right)^{3}}{3 !} y^{(5)}\left(x_{n}\right)+\frac{\left(\frac{15}{6} h\right)^{4}}{4 !} y^{(6)}\left(x_{n}\right)+\frac{\left(\frac{15}{6} h\right)^{5}}{5 !} y^{(7)}\left(x_{n}\right) \\
& \left.+\frac{\left(\frac{15}{6} h\right)^{6}}{6 !} y^{(8)}\left(x_{n}\right)\right)+\phi_{14}\left(y^{(2)}\left(x_{n}\right)+\frac{20}{6} h y^{(3)}\left(x_{n}\right)+\frac{\left(\frac{20}{6} h\right)^{2}}{2 !} y^{(4)}\left(x_{n}\right)+\frac{\left(\frac{20}{6} h\right)^{3}}{3 !} y^{(5)}\left(x_{n}\right)\right. \\
& \left.+\frac{\left(\frac{20}{6} h\right)^{4}}{4 !} y^{(6)}\left(x_{n}\right)+\frac{\left(\frac{20}{6} h\right)^{5}}{5 !} y^{(7)}\left(x_{n}\right)+\frac{\left(\frac{20}{6} h\right)^{6}}{6 !} y^{(8)}\left(x_{n}\right)\right)+\phi_{15}\left(y^{(2)}\left(x_{n}\right)+\frac{25}{6} h y^{(3)}\left(x_{n}\right)\right. \\
& \left.+\frac{\left(\frac{25}{6} h\right)^{2}}{2 !} y^{(4)}\left(x_{n}\right)+\frac{\left(\frac{25}{6} h\right)^{3}}{3 !} y^{(5)}\left(x_{n}\right)+\frac{\left(\frac{25}{6} h\right)^{4}}{4 !} y^{(6)}\left(x_{n}\right)+\frac{\left(\frac{25}{6} h\right)^{5}}{5 !} y^{(7)}\left(x_{n}\right)+\frac{\left(\frac{25}{6} h\right)^{6}}{6 !} y^{(8)}\left(x_{n}\right)\right) \\
& +\phi_{16}\left(y^{(2)}\left(x_{n}\right)+5 h y^{(3)}\left(x_{n}\right)+\frac{(5 h)^{2}}{2 !} y^{(4)}\left(x_{n}\right)+\frac{(5 h)^{3}}{3 !} y^{(5)}\left(x_{n}\right)+\frac{(5 h)^{4}}{4 !} y^{(6)}\left(x_{n}\right)\right. \\
& \left.\left.+\frac{(5 h)^{5}}{5 !} y^{(7)}\left(x_{n}\right)+\frac{(5 h)^{6}}{6 !} y^{(8)}\left(x_{n}\right)\right)\right\}
\end{aligned}
$$

This expression as seen in (Lambert, 1973) was limited to the derivation of multistep schemes for first order ordinary differential equations. Therefore, since this article is dealing with higher order ordinary differential equations, the Taylor expansion for $y_{n+a}^{(n)}=y^{(n)}\left(x_{n}+a h\right)$ about $x_{n}$ is needed and defined as:

$$
\begin{aligned}
y^{(n)}\left(x_{n}+a h\right)= & y^{(n)}\left(x_{n}\right)+a h y^{(n+1)}\left(x_{n}\right) \\
& +\frac{(a h)^{2}}{2 !} y^{(n+2)}\left(x_{n}\right)+\cdots
\end{aligned}
$$

Equation 4 and 5 are used for the expansion of $y_{n+\frac{5}{6} \xi}, f_{n+\frac{5}{6} i}$ and $y_{n+\frac{5}{6} \xi}^{\prime}$ where $y_{n+a h}=f\left(x_{n}+a h\right)+y^{\prime \prime}\left(x_{n}+\right.$ $a h)$. The obtained expressions are then substituted back to Equation 2 and 3 to obtain the required scheme, however the truncation of the Taylor series expansion is with respect to the number of unknown $\phi_{\xi i}$ or $\omega_{\xi i}$.

For better clarification, consider Equation 2 with $\xi=$ 1 which gives:

$$
y_{n+\frac{5}{6}}=\sum_{i=0}^{1} \frac{\left(\frac{5}{6} h\right)^{i}}{i !} y_{n}^{(i)}+h^{2} \sum_{i=0}^{6} \phi_{1 i} f_{n+\frac{5}{6} i}
$$

This is equivalent to the expression: 
Equating coefficients of $h^{m} y^{(m)}\left(x_{n}\right)$ and rewriting in matrix form $A x=B$, Equation 7 takes the form:

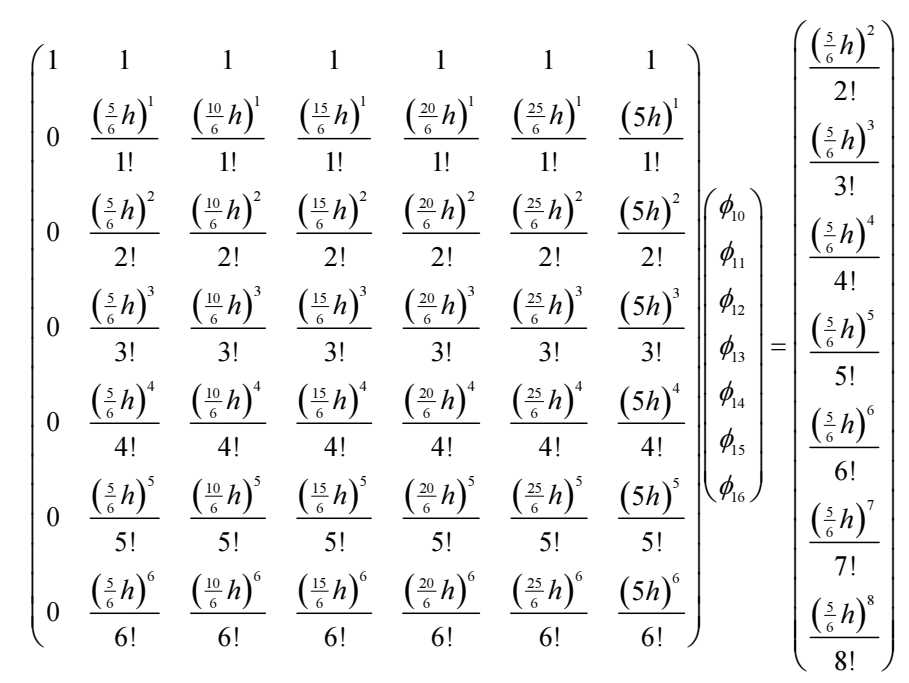

Using matrix inverse formula for system of linear equations, the value of the unknown coefficients are obtained to be:

$$
\begin{aligned}
& \left(\phi_{10}, \phi_{11}, \phi_{12}, \phi_{13}, \phi_{14}, \phi_{15}, \phi_{16}\right)^{T} \\
& =\left(\frac{142745}{870912}, \frac{6875}{20736},-\frac{28585}{96768}, \frac{53105}{217728},-\frac{38515}{290304}, \frac{2015}{48384},-\frac{4975}{870912}\right)
\end{aligned}
$$

Following the same approach, the remaining unknown coefficients are obtained to be:

$$
\begin{aligned}
& \left(\phi_{20}, \phi_{21}, \phi_{22}, \phi_{23}, \phi_{24}, \phi_{25}, \phi_{26}\right)^{T} \\
& =\left(\frac{5135}{13608}, \frac{485}{378},-\frac{50}{81}, \frac{985}{1701},-\frac{485}{1512}, \frac{115}{1134},-\frac{95}{6804}\right) \\
& \left(\phi_{30}, \phi_{31}, \phi_{32}, \phi_{33}, \phi_{34}, \phi_{35}, \phi_{36}\right)^{T} \\
& =\left(\frac{6325}{10752}, \frac{4125}{1792},-\frac{1335}{3584}, \frac{125}{128},-\frac{1815}{3584}, \frac{285}{1792},-\frac{235}{10752}\right) \\
& \left(\phi_{40}, \phi_{41}, \phi_{42}, \phi_{43}, \phi_{44}, \phi_{45}, \phi_{46}\right)^{T} \\
& =\left(\frac{1360}{1701}, \frac{1880}{567},-\frac{10}{189}, \frac{3280}{1701},-\frac{50}{81}, \frac{40}{189},-\frac{50}{1701}\right) \\
& \left(\phi_{50}, \phi_{51}, \phi_{52}, \phi_{53}, \phi_{54}, \phi_{55}, \phi_{56}\right)^{T} \\
& =\left(\frac{880625}{870912}, \frac{209375}{48384}, \frac{78125}{290304}, \frac{640625}{217728},-\frac{15625}{96768}, \frac{6875}{20736},-\frac{34375}{870912}\right) \\
& \left(\phi_{60}, \phi_{61}, \phi_{62}, \phi_{63}, \phi_{64}, \phi_{65}, \phi_{66}\right)^{T} \\
& =\left(\frac{205}{168}, \frac{75}{14}, \frac{15}{28}, \frac{85}{21}, \frac{15}{56}, \frac{15}{14}, 0\right) \\
& \left(\omega_{10}, \omega_{11}, \omega_{12}, \omega_{13}, \omega_{14}, \omega_{15}, \omega_{16}\right)^{T} \\
& =\left(\frac{19087}{72576}, \frac{2713}{3024},-\frac{15487}{24192}, \frac{293}{567},-\frac{6737}{24192}, \frac{263}{3024},-\frac{863}{72576}\right) \\
& \left(\omega_{20}, \omega_{21}, \omega_{22}, \omega_{23}, \omega_{24}, \omega_{25}, \omega_{26}\right)^{T} \\
& =\left(\frac{1139}{4536}, \frac{235}{189}, \frac{11}{1512}, \frac{166}{567},-\frac{269}{1512}, \frac{11}{189},-\frac{37}{4536}\right) \\
& \left(\omega_{30}, \omega_{31}, \omega_{32}, \omega_{33}, \omega_{34}, \omega_{35}, \omega_{36}\right)^{T} \\
& =\left(\frac{685}{2688}, \frac{135}{112}, \frac{387}{896}, \frac{17}{21},-\frac{243}{896}, \frac{9}{112},-\frac{29}{2688}\right) \\
& \left(\omega_{40}, \omega_{41}, \omega_{42}, \omega_{43}, \omega_{44}, \omega_{45}, \omega_{46}\right)^{T} \\
& =\left(\frac{143}{567}, \frac{232}{189}, \frac{64}{189}, \frac{752}{567}, \frac{29}{189}, \frac{8}{189},-\frac{4}{567}\right) \\
&
\end{aligned}
$$

$\left(\omega_{50}, \omega_{51}, \omega_{52}, \omega_{53}, \omega_{54}, \omega_{55}, \omega_{56}\right)^{T}$

$=\left(\frac{18575}{72576}, \frac{3625}{3024}, \frac{10625}{24112}, \frac{625}{567}, \frac{19375}{24192}, \frac{1175}{3024},-\frac{1375}{72576}\right)$

$\left(\omega_{60}, \omega_{61}, \omega_{62}, \omega_{63}, \omega_{64}, \omega_{65}, \omega_{66}\right)^{T}$

$=\left(\frac{41}{168}, \frac{9}{7}, \frac{9}{56}, \frac{34}{21}, \frac{9}{56}, \frac{9}{7}, \frac{41}{168}\right)$

Substituting all the obtained coefficients back in Equations 2 and 3 gives the hybrid block schemes:

$$
\begin{aligned}
& y_{n+\frac{5}{6}}=y_{n}+\frac{5}{6} h y_{n}^{\prime} \\
& +\frac{h^{2}}{870912}\left[\begin{array}{l}
142745 f_{n}+288750 f_{n+\frac{5}{6}}-257265 f_{n+\frac{10}{6}}+212420 f_{n+\frac{15}{6}} \\
-115545 f_{n+\frac{20}{6}}+36270 f_{n+\frac{23}{6}}-4975 f_{n+5}
\end{array}\right] \\
& y_{n+\frac{10}{6}}=y_{n}+\frac{10}{6} h y_{n}^{\prime} \\
& +\frac{h^{2}}{13608}\left[\begin{array}{l}
5135 f_{n}+17460 f_{n+\frac{5}{6}}-8400 f_{n+\frac{10}{6}}+7880 f_{n+\frac{15}{6}} \\
-4365 f_{n+\frac{20}{6}}+1380 f_{n+\frac{25}{6}}-190 f_{n+5}
\end{array}\right] \\
& y_{n+\frac{15}{6}}=y_{n}+\frac{15}{6} h y_{n}^{\prime} \\
& +\frac{h^{2}}{10752}\left[\begin{array}{l}
6325 f_{n}+24750 f_{n+\frac{5}{6}}-4005 f_{n+\frac{10}{6}}+10500 f_{n+\frac{15}{6}} \\
-5445 f_{n+\frac{20}{6}}+1710 f_{n+\frac{25}{6}}-235 f_{n+5}
\end{array}\right] \\
& y_{n+\frac{20}{6}}=y_{n}+\frac{20}{6} h y_{n}^{\prime} \\
& +\frac{h^{2}}{1701}\left[\begin{array}{l}
1360 f_{n}+5640 f_{n+\frac{5}{6}}-90 f_{n+\frac{10}{6}}+3280 f_{n+\frac{15}{6}} \\
-1050 f_{n+\frac{20}{6}}+360 f_{n+\frac{25}{6}}-50 f_{n+5}
\end{array}\right] \\
& y_{n+\frac{25}{6}}=y_{n}+\frac{25}{6} h y_{n}^{\prime} \\
& +\frac{h^{2}}{1701}\left[\begin{array}{l}
880625 f_{n}+3768750 f_{n+\frac{5}{6}}+234375 f_{n+\frac{10}{6}}+2562500 f_{n+\frac{15}{6}} \\
-140625 f_{n+\frac{20}{6}}+288750 f_{n+\frac{25}{6}}-34375 f_{n+5}
\end{array}\right] \\
& y_{n+5}=y_{n}+5 h y_{n}^{\prime}+\frac{h^{2}}{168}\left[\begin{array}{l}
205 f_{n}+900 f_{n+\frac{5}{6}}+90 f_{n+\frac{10}{6}} \\
+680 f_{n+\frac{15}{6}}+45 f_{n+\frac{20}{6}}+180 f_{n+\frac{25}{6}}
\end{array}\right]
\end{aligned}
$$


Together with its derivatives:

$$
\begin{aligned}
& y_{n+\frac{5}{6}}^{\prime}=y_{n}^{\prime}+\frac{h}{870912}\left[\begin{array}{l}
19087 f_{n}+65112 f_{n+\frac{5}{6}}-46461 f_{n+\frac{11}{6}}+37504 f_{n+\frac{15}{6}} \\
-20211 f_{n+\frac{3}{6}}+6312 f_{n+\frac{35}{6}}-863 f_{n+5}
\end{array}\right] \\
& y_{n+\frac{10}{6}}^{\prime}=y_{n}^{\prime}+\frac{h}{4536}\left[\begin{array}{l}
1139 f_{n}+5640 f_{n+\frac{5}{6}}+33 f_{n+\frac{10}{6}}+1328 f_{n+\frac{15}{6}} \\
-807 f_{n+\frac{20}{6}}+264 f_{n+\frac{25}{6}}-37 f_{n+5}
\end{array}\right] \\
& y_{n+\frac{15}{6}}^{\prime}=y_{n}^{\prime}+\frac{h}{2688}\left[\begin{array}{l}
685 f_{n}+3240 f_{n+\frac{5}{6}}+1161 f_{n+\frac{10}{6}}+2176 f_{n+\frac{15}{6}} \\
-729 f_{n+\frac{20}{6}}+216 f_{n+\frac{25}{6}}-29 f_{n+5}
\end{array}\right] \\
& y_{n+\frac{20}{6}}^{\prime}=y_{n}^{\prime}+\frac{h}{567}\left[\begin{array}{l}
143 f_{n}+696 f_{n+\frac{5}{6}}+192 f_{n+\frac{10}{6}}+752 f_{n+\frac{15}{6}} \\
+87 f_{n+\frac{20}{6}}+24 f_{n+\frac{25}{6}}-4 f_{n+5}
\end{array}\right] \\
& y_{n+\frac{25}{6}}^{\prime}=y_{n}^{\prime}+\frac{h}{72576}\left[\begin{array}{l}
18575 f_{n}+87000 f_{n+\frac{5}{6}}+31875 f_{n+\frac{10}{6}} \\
+80000 f_{n+\frac{15}{6}}+58125 f_{n+\frac{20}{6}} \\
+28200 f_{n+\frac{25}{6}}-1375 f_{n+5}
\end{array}\right] \\
& y_{n+5}^{\prime}=y_{n}^{\prime}+\frac{h}{168}\left[\begin{array}{l}
41 f_{n}+216 f_{n+\frac{5}{6}}+27 f_{n+\frac{10}{6}}+272 f_{n+\frac{15}{6}} \\
+27 f_{n+\frac{20}{6}}+216 f_{n+\frac{25}{6}}+41 f_{n+5}
\end{array}\right]
\end{aligned}
$$

\section{Results}

\section{Properties of the Method}

\section{Zero Stability}

The hybrid block method is said to be zero-stable if the roots $z$ of the first characteristic polynomial $\rho(z)=$ det $\left(z A^{0}-A^{1}\right)$ satisfies $|z| \leq 1$ and the root $|z|=1$ has multiplicity not greater than the order of the differential equation (Kuboye and Omar, 2015). Therefore, the first characteristic polynomial of the hybrid block method (8) is obtained as:

$$
\operatorname{det}\left(z A^{0}-A^{1}\right)=\left(\begin{array}{llllll}
1 & 0 & 0 & 0 & 0 & 0 \\
0 & 1 & 0 & 0 & 0 & 0 \\
0 & 0 & 1 & 0 & 0 & 0 \\
0 & 0 & 0 & 1 & 0 & 0 \\
0 & 0 & 0 & 0 & 1 & 0 \\
0 & 0 & 0 & 0 & 0 & 1
\end{array}\right)-\left(\begin{array}{cccccc}
0 & 0 & 0 & 0 & 0 & 1 \\
0 & 0 & 0 & 0 & 0 & 1 \\
0 & 0 & 0 & 0 & 0 & 1 \\
0 & 0 & 0 & 0 & 0 & 1 \\
0 & 0 & 0 & 0 & 0 & 1 \\
0 & 0 & 0 & 0 & 0 & 1
\end{array}\right) \mid
$$

Hence the roots of the polynomial are $z=0,0,0,0,0,1$. Thus, the hybrid block method is zero-stable.

\section{Order of the Method}

Rewrite the hybrid block method in Equation 2 in its corresponding linear difference operator form as defined below:

$$
\begin{aligned}
& L\{y(x): h\}=y_{n+\frac{5}{6}} \\
& -\sum_{i=0}^{1} \frac{\left(\frac{5}{6} \xi h\right)^{i}}{i !} y_{n}^{(i)}-h^{2} \sum_{i=0}^{6} \phi_{\xi i} f_{n+\frac{5}{6} i}, \xi=1,2, \cdots, 6
\end{aligned}
$$

Following the conventional approach for obtaining the order of a linear multistep method from (Lambert, 1973), the individual terms in (8) are expanded using Taylor series expansion about $x_{n}$ as defined in Equation 4. After much simplification, the hybrid block schemes in (8) are found to be of order $[7,7,7,7,7,7]^{T}$ with error constant of:

$$
\left[\begin{array}{l}
1.28841 \times 10^{-3} \\
3.18568 \times 10^{-3} \\
4.98360 \times 10^{-3} \\
6.78153 \times 10^{-3} \\
8.67879 \times 10^{-3} \\
9.96720 \times 10^{-3}
\end{array}\right]
$$

\section{Consistency}

The hybrid block method is said to be consistent if it has an order more than or equal to one (Abdelrahim and Omar, 2016). Therefore, our method is consistent.

\section{Convergence}

The sufficient conditions for a linear multistep method to be convergent are zero stability and consistency (Henrici, 1963). Therefore since the hybrid block method is zero-stable and consistent, hence it is convergent.

\section{Numerical Experiments}

The following second order initial value problems were numerically solved for comparison of accuracy in terms of error with previously existing methods:

\section{Problem 1:}

$$
y^{\prime \prime}=-y+2 \cos x, y(0)=1, y^{\prime}(0)=0,0 \leq x \leq 1
$$

\section{Exact Solution:}

$$
y(x)=\cos x+x \sin x
$$

Problem 2:

$$
y^{\prime \prime}=y, y(0)=1, y^{\prime}(0)=1,0 \leq x \leq 1
$$

\section{Exact Solution:}

$$
y(x)=e^{x}
$$

Problems 1 and 2 above were solved by (Kuboye and Omar, 2015) using a non-hybrid six-step block method of order seven and the maximum errors within the given 
interval were selected. The hybrid block method was adopted to numerically solve both problems and the results obtained are compared as shown in Table 1.

Problem 3:

$$
y^{\prime \prime}=y^{\prime}, y(0)=0, y^{\prime}(0)=-1,0 \leq x \leq 1
$$

Exact Solution:

$$
y(x)=1-e^{x}
$$

Problem 3 was solved by (Mohammed et al., 2010) using a six-step block method of order seven. Comparison is made between their method and the new hybrid block as displayed in Table 2 .

Problem 4:

$$
y^{\prime \prime}=-y, y(0)=1, y^{\prime}(0)=1
$$

\section{Exact Solution:}

$$
y(x)=\cos x+\sin x
$$

Awari (2013) computed the numerical solution of Problem 4 and the results with comparison to the new hybrid method is shown in Table 3.

\section{Problem 5:}

$$
y^{\prime \prime}-4 y^{\prime}+8 y=x^{3}, y(0)=2, y^{\prime}(0)=4
$$

\section{Exact Solution:}

$$
y(x)=\frac{3}{32} x+2(\cos 2 x) e^{2 x}-\frac{3}{64} e^{2 x} \sin 2 x+\frac{3}{16} x^{2}+\frac{1}{8} x^{3}
$$

Problem 5 was solved using the new hybrid block in Equation 8 and comparison in terms of error was made

\begin{tabular}{|c|c|c|c|c|}
\hline$x$ & Exact solution & Computed solution & $\begin{array}{l}\text { Error } \\
\text { (Mohammed et al., 2010) }\end{array}$ & $\begin{array}{l}\text { Error (Hybrid } \\
\text { block method) }\end{array}$ \\
\hline 0.0833 & -0.08690404952123 & -0.08690404951952 & - & $1.705053 \mathrm{E}-12$ \\
\hline 0.1000 & -0.10517091800000 & - & $5.7260 \mathrm{E}-06$ & - \\
\hline 0.1667 & -0.18136041286565 & -0.18136041286124 & - & 4.405920E-12 \\
\hline 0.2000 & -0.22140275800000 & - & $6.6391 \mathrm{E}-06$ & - \\
\hline 0.2500 & -0.28402541668774 & -0.28402541668054 & - & $7.198242 \mathrm{E}-12$ \\
\hline 0.3000 & -0.34985880800000 & - & 7.0283E-06 & - \\
\hline 0.3333 & -0.39561242508609 & -0.39561242507585 & - & $1.024042 \mathrm{E}-11$ \\
\hline 0.4000 & -0.49182469800000 & - & $7.4539 \mathrm{E}-06$ & - \\
\hline 0.4167 & -0.51689679638821 & -0.51689679637454 & - & $1.367073 \mathrm{E}-11$ \\
\hline 0.5000 & -0.64872127070013 & -0.64872127068352 & $7.8935 \mathrm{E}-06$ & $1.661205 \mathrm{E}-11$ \\
\hline 0.5833 & -0.79200182565576 & -0.79200182563489 & - & $2.086653 \mathrm{E}-11$ \\
\hline 0.6000 & -0.82211880000000 & - & $8.1942 \mathrm{E}-06$ & - \\
\hline 0.6667 & -0.94773404105468 & -0.94773404102779 & - & $2.688882 \mathrm{E}-11$ \\
\hline 0.7000 & -1.01375270000000 & - & $8.1810 \mathrm{E}-06$ & - \\
\hline 0.7500 & -1.11700001661267 & -1.11700001657948 & - & 3.319833E-11 \\
\hline 0.8000 & -1.22554092800000 & - & $8.1810 \mathrm{E}-06$ & - \\
\hline 0.8333 & -1.30097589089283 & -1.30097589085276 & - & 4.006750E-11 \\
\hline 0.9000 & -1.45960311100000 & - & $8.1730 \mathrm{E}-06$ & - \\
\hline 0.9167 & -1.50094001366213 & -1.50094001361439 & - & $4.773781 \mathrm{E}-11$ \\
\hline 1.0000 & -1.71828182800000 & -1.71828182840427 & $8.1650 \mathrm{E}-06$ & 5.477729E-11 \\
\hline
\end{tabular}
with (Abdelrahim and Omar, 2016). Results are as displayed in Table 4.

Table 1. Comparison between new hybrid block method and (Adesanya et al., 2012) for Problem 1 and Problem 2

\begin{tabular}{lll}
\hline Problem 1 & & \\
$h$ & Error (Kuboye and Omar, 2015) & Error (Hybrid block method) \\
0.01 & $1.428607 \mathrm{E}-11$ & $8.881784 \mathrm{E}-16$ \\
0.001 & $1.687539 \mathrm{E}-13$ & $2.220446 \mathrm{E}-15$ \\
Problem 2 & & \\
$h$ & Error (Kuboye and Omar, 2015) & Error (Hybrid block method) \\
0.01 & $1.428607 \mathrm{E}-11$ & $8.881784 \mathrm{E}-16$ \\
0.001 & $1.687539 \mathrm{E}-13$ & $2.220446 \mathrm{E}-15$ \\
\hline
\end{tabular}

Table 2. Comparison between new hybrid block method and (Mohammed et al., 2010) for Problem 3 
Table 3. Comparison between new hybrid block method and (Kayode and Adeyeye, 2011) for Problem 4

\begin{tabular}{|c|c|c|c|c|}
\hline$x$ & Exact solution & Computed solution & $\begin{array}{l}\text { Error (Kayode and } \\
\text { Adeyeye, 2011) }\end{array}$ & $\begin{array}{l}\text { Error (Hybrid } \\
\text { block method) }\end{array}$ \\
\hline 0.0833 & 1.0797667029008697 & 1.0797667028998994 & - & $9.703349 \mathrm{E}-13$ \\
\hline 0.1000 & 1.0948375819000000 & - & $1.157 \mathrm{E}-7$ & - \\
\hline 0.1667 & 1.1520393642563400 & 1.1520393642539457 & - & $2.394307 \mathrm{E}-12$ \\
\hline 0.2000 & 1.1787359086000000 & - & $3.099 \mathrm{E}-7$ & - \\
\hline 0.2500 & 1.2163163809651676 & 1.2163163809614408 & - & $3.726797 \mathrm{E}-12$ \\
\hline 0.3000 & 1.2508566958000000 & - & $5.055 \mathrm{E}-7$ & - \\
\hline 0.3333 & 1.2721516431108899 & 1.2721516431058548 & - & $5.035083 \mathrm{E}-12$ \\
\hline 0.4000 & 1.3104793363000000 & - & $6.957 \mathrm{E}-7$ & - \\
\hline 0.4167 & 1.3191576301549550 & 1.3191576301485788 & - & $6.376233 \mathrm{E}-12$ \\
\hline 0.5000 & 1.3570081004945758 & 1.3570081004873182 & $8.789 \mathrm{E}-7$ & $7.257528 \mathrm{E}-12$ \\
\hline 0.5833 & 1.3854403557186268 & 1.3854403557113075 & - & $7.319256 \mathrm{E}-12$ \\
\hline 0.6000 & 1.3899780883000000 & - & $1.054 \mathrm{E}-6$ & - \\
\hline 0.6667 & 1.4042570638466851 & 1.4042570638392982 & - & $7.386980 \mathrm{E}-12$ \\
\hline 0.7000 & 1.4090598745000000 & - & $1.008 \mathrm{E}-6$ & - \\
\hline 0.7500 & 1.4133276288971550 & 1.4133276288897612 & - & $7.393863 \mathrm{E}-12$ \\
\hline 0.8000 & 1.4140628003000000 & - & $9.226 \mathrm{E}-7$ & - \\
\hline 0.8333 & 1.4125890972790938 & 1.4125890972717421 & - & $7.351675 \mathrm{E}-12$ \\
\hline 0.9000 & 1.4049368779000000 & - & $8.261 \mathrm{E}-7$ & - \\
\hline 0.9167 & 1.4020465947169871 & 1.4020465947097280 & - & $7.259082 \mathrm{E}-12$ \\
\hline 1.0000 & 1.3817732906760363 & 1.3817732906689242 & $7.216 \mathrm{E}-7$ & $7.112089 \mathrm{E}-12$ \\
\hline
\end{tabular}

Table 4. Comparison between new hybrid block method and (Abdelrahim and Omar, 2016) for Problem 5

\begin{tabular}{|c|c|c|c|c|}
\hline$x$ & Exact solution & Computed solution & $\begin{array}{l}\text { Error (Abdelrahim and } \\
\text { Omar, 2016) }\end{array}$ & $\begin{array}{l}\text { Error (Hybrid } \\
\text { block method) }\end{array}$ \\
\hline 0.0833 & 2.3299813627353112 & 2.3299813454790241 & - & $1.725629 \mathrm{E}-08$ \\
\hline 0.1000 & 2.3941125770000000 & - & $5.7260 \mathrm{E}-06$ & - \\
\hline 0.1667 & 2.6375944892958487 & 2.6375944390018171 & - & 5.029403E-08 \\
\hline 0.2000 & 2.7481413330000000 & - & $6.6391 \mathrm{E}-06$ & - \\
\hline 0.2500 & 2.8938356161427006 & 2.8938355248284888 & - & $9.131421 \mathrm{E}-08$ \\
\hline 0.3000 & 3.0078669400000000 & - & 7.0283E-06 & - \\
\hline 0.3333 & 3.0616545199215750 & 3.0616543769812110 & - & $1.429404 \mathrm{E}-07$ \\
\hline 0.4000 & 3.1017624050000000 & - & 7.4539E-06 & - \\
\hline 0.4167 & 3.0952313771655420 & 3.0952311710464442 & - & 2.061191E-07 \\
\hline 0.5000 & 3.1017624050000000 & 2.9395428239868138 & $7.8935 \mathrm{E}-06$ & $2.767584 \mathrm{E}-07$ \\
\hline
\end{tabular}

\section{Discussion}

This article has considered certain second order initial problems as highlighted in Problems 1-5. The new hybrid block method was adopted to obtain numerical approximations to these problems. As displayed in Tables 1-4, more accurate results in comparison to the exact solution is displayed by the new hybrid block method.

\section{Conclusion}

This article has introduced a new hybrid block method for the numerical approximation of second order initial value problems. To validate the superiority of the new hybrid block method, certain numerical examples were considered which have been solved previously in literature and comparison was made in terms of absolute error, that is, the difference between the exact and computed solution. The results in the tables above show improved accuracy and hence making this new hybrid method suitable for application to obtain the approximate numerical solution of real life problems modeled in the form of Equation 1 above.

\section{Acknowledgment}

The authors gratefully acknowledge the support of Research and Innovation Centre (RIMC), Universiti Utara Malaysia in helping us in terms of administrative work for the purpose of publishing this article. Article publication funded by Universiti Utara Malaysia covering the costs to publish in open access.

\section{Funding Information}

Article publication was funded by Universiti Utara Malaysia.

\section{Author's Contributions}

Both authors contributed equally to the work. 


\section{Ethics}

The authors declare no conflict of interest.

\section{References}

Abdelrahim, R.F. and Z. Omar, 2016. Direct solution of second-order ordinary differential equation using a single-step hybrid block method of order five. Math. Comput. Applic., 21: 1-7. DOI: $10.3390 / \mathrm{mca} 21020012$

Abdul Majid, Z., N.Z. Mokhtar and M. Suleiman, 2012. Direct two-point block one-step method for solving general second-order ordinary differential equations. Math. Prob. Eng. DOI: 10.1155/2012/184253

Adesanya, A.O., M.R. Odekunle and A.O. Adeyeye, 2012. Continuous block hybrid predictor corrector method for the solution of $y^{\prime \prime}=f\left(x, y, y^{\prime}\right)$. Int. J. Math. Soft Comput., 2: 35-42.

Awari, Y.S., 2013. Derivation and application of sixpoint linear multistep numerical method for solution of second order initial value problems. IOSR J. Math., 7: 23-29.

Henrici, P., 1963. Some applications of the quotientdifference algorithm. Proc. Symp. Applied Math., 15: 159-183.

James, A., A.O. Adesanya and S. Joshua, 2013. Continuous block method for the solution of second order initial value problems of ordinary differential equation. Int. J. Pure Applied Math., 83: 405-416.

Jator, S.N., 2007. A sixth order linear multistep method for direct solution of second order ordinary differential equations. Int. J. Pure Applied Math., 4: 457-472.
Kayode, S.J. and O. Adeyeye, 2011. A 3-step hybrid method for direct solution of second order initial value problems. Aus. J. Basic Applied Sci., 5: 2121-2126.

Kuboye, J.O. and Z. Omar, 2015. Derivation of a sixstep block method for direct solutions of second order ordinary differential equations. Math. Comput. Applic., 20: 151-159. DOI: 10.19029/mca-2015-013

Lambert, J.D., 1973. Computational Methods in Ordinary Differential Equations. 1st Edn., Wiley, London, ISBN-10: 0471511943, pp: 278.

Moaddy, K., M. AL-Smadi and I. Hashim, 2015. A novel representation of the exact solution for differential algebraic equations system using residual power-series method. Discrete Dynam. Nature Society. DOI: 10.1155/2015/205207

Mohammed, U., M. Jiya and A.A. Mohammed, 2010. A class of six step block method for solution of general second order ordinary differential equations. Pacific J. Sci. Technol., 11: 273-277.

Omar, Z. and M.B. Suleiman, 2016. Parallel two-point explicit block method for solving high-order ordinary differential equations. Int. J. Simul. Process Modell., 2: 227-231.

DOI: 10.1504/IJSPM.2006.012650

See, P.P., Z.A. Majid and M. Suleiman, 2016. Solving nonlinear two point boundary value problem using two step direct method. J. Q. Measure. Anal., 7: 127-138. 